\title{
Plataforma para apoio a modelagem de próteses com base em Processamento Digital de Imagens e Deep Learning
}

\author{
Bianka Tallita Passos \\ Universidade do Vale do Itajaí - \\ UNIVALI \\ Itajaí, Santa Catarina, BR \\ biankatpas@edu.univali.br
}

\author{
Moira Cristina Cubas Fatiga \\ Tillmann \\ Universidade do Vale do Itajaí - \\ UNIVALI \\ Itajaí, Santa Catarina, BR \\ moira.fatiga@edu.univali.br
}

\author{
Anita Maria da Rocha \\ Fernandes \\ Universidade do Vale do Itajaí - \\ UNIVALI \\ Itajaí, Santa Catarina, BR \\ anita.fernandes@univali.br
}

\begin{abstract}
Medical practice in general, and dentistry in particular, generates data sources, such as high-resolution medical images and electronic medical records. Digital image processing algorithms take advantage of the datasets, enabling the development of dental applications such as tooth, caries, crown, prosthetic, dental implant, and endodontic treatment detection, as well as image classification. The goal of image classification is to comprehend it as a whole and classify the image by assigning it to a specific label. This work presents the proposal of a tool that helps the dental prosthesis specialist to exchange information with the laboratory. The proposed solution uses deep learning to classify image, in order to improve the understanding of the structure required for modeling the prosthesis. The image database used has a total of 1215 images. Of these, 60 were separated for testing. The prototype achieved $98.33 \%$ accuracy.
\end{abstract}

\section{KEYWORDS}

Redes Neurais Convolucionais, Processamento Digital de Imagens, Prótese Dental

\section{INTRODUÇÃO}

A reabilitação oral consiste em um conjunto de técnicas e tratamentos que têm por finalidade restabelecer o aspecto funcional do sorriso, garantindo que o paciente recupere sua autoestima e restabeleça as funções dentárias primordiais. A prótese dentária restaura os dentes perdidos e impacta diretamente no tratamento de disfunções bucais [1].

A sequência da modelagem da prótese dentária é baseada em um planejamento minucioso, feito pelo cirurgião dentista. De acordo com o grau de comprometimento dentário de cada paciente, o profissional define o tipo de reabilitação oral que necessita ser realizada. O tratamento é conduzido com base em exames prévios solicitados pelo cirurgião dentista, como raios-x, tomografia da face, exames clínicos, entre outros [2].

O desenvolvimento da prótese gera fontes de dados, como imagens de alta resolução e registros eletrônicos que possibilitam o desenvolvimento de soluções baseadas em processamento digital de imagens para apoiar o processo de reabilitação oral. Dentre as soluções baseadas em processamento digital de imagens, podemos destacar o uso de técnicas de detecção de objetos para identificar e numerar dentes, bem como identificar demais elementos que impactam a modelagem da prótese.

\section{TRABALHOS RELACIONADOS}

Dentre as técnicas de reconhecimento de objetos em imagens, destacam-se a extração de características e algoritmos de aprendizado de máquina, bem como aprendizagem profunda. Os trabalhos mais recentes disponíveis na literatura, são baseados principalmente no uso redes neurais convolucionais ( $\mathrm{CNN}$ do inglês Convolutional Neural network).

Como exemplo, Hiraiwa et al. [3] propuseram uma solução utilizando CNN para classificar a morfologia radicular de primeiros molares inferiores em radiografias panorâmicas. O objetivo da pesquisa era efetuar a diferenciação das raízes. Imagens de tomografias e radiografias panorâmicas de 760 primeiros molares inferiores de 400 pacientes, não submetidos a tratamento de canal, foram analisadas através do protótipo. Os procedimentos de treinamento e testes foram realizados utilizando as arquiteturas AlexNet e GoogleNet.

Krois et al. [4] utilizaram redes neurais para detectar perda óssea periodontal em radiografias panorâmicas. Durante a fase de desenvolvimento do protótipo, os autores efetuaram testes com CNNs pré-treinadas, como a VGG16 e Inception.v3. No entanto, essas arquiteturas causavam sobreajuste e reduziam a capacidade de discriminação. O modelo final proposto foi uma nova rede neural profunda. A arquitetura proposta pelos autores possui sete camadas, sendo parametrizada por um número total de 4.299 .651 pesos. Para comparação, seis dentistas avaliaram os segmentos de imagem para perda óssea periodontal. Os esforços diagnósticos dos dentistas ao usar radiografias podem ser reduzidos pela aplicação de tecnologias baseadas em aprendizado de máquina.

Tuzoff et al. [5] utilizaram a arquitetura de rede Faster R-CNN na detecção e numeração dentária de radiografias panorâmicas. Os autores utilizaram um conjunto de dados contendo 1352 radiografias panorâmicas de adultos, escolhidas aleatoriamente, para treinar a rede neural. Para a tarefa de detecção de dentes, o algoritmo atingiu as seguintes métricas de desempenho: uma sensibilidade de 0,9941 e uma precisão de 0,9945. Para a numeração dos dentes, a sensibilidade foi 0,9800 e a especificidade 0,9994 .

\section{SOLUÇÃO PROPOSTA}

Este trabalho propõe o uso de algoritmos de aprendizado profundo para identificar a qual classe a imagem pertence, bem como detectar e reconhecer os objetos que são importantes para contribuir com o tratamento de prótese total dos pacientes. A proposta de uma solução utilizando CNN é justificada pelos trabalhos mais recentes encontrados na literatura, que são baseadas principalmente no uso de aprendizado de máquina e redes neurais artificiais [6]. 
Seguindo o exposto em trabalhos correlatos, será feito um levantamento de bases de imagens públicas. As bases encontradas serão validadas com especialistas da área de prótese dentária. De posse da base de dados, os objetos a serem reconhecidos serão identificados, o que permitirá a extração de características para efetuar o treinamento da rede neural convolucional. Do total de imagens, $70 \%$ serão utilizadas para treinamento, $10 \%$ para validação e $20 \%$ para testes.

A base de treinamento é utilizada para que a rede aprenda, a de validação é responsável por fornecer uma avaliação imparcial do modelo no conjunto de dados de treinamento enquanto ajusta os hiperparâmetros do modelo e a base de testes por avaliar o modelo gerado, portanto só é utilizada quando o treinamento finaliza. $\mathrm{O}$ fluxograma da solução proposta é apresentado na Figura 1.

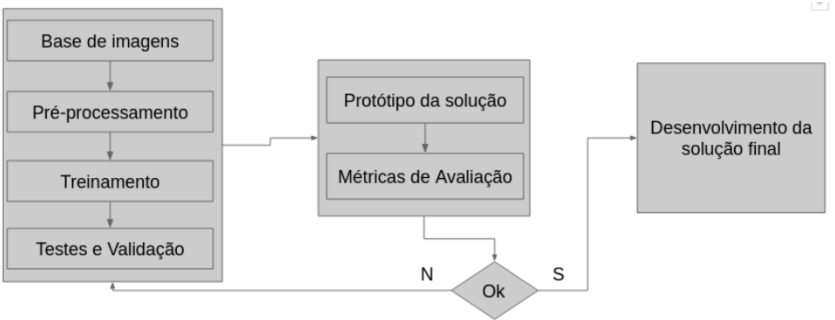

Figura 1: Fluxograma da solução proposta.

O trabalho está em desenvolvimento, e até o momento foi efetuado o treinamento de uma rede neural convolucional - da arquitetura Inception - por 5 mil épocas, para inferir a qual classe pertence a imagem do paciente, a saber: panorâmica, foto de perfil, telerradiografia lateral, foto intrabucal direita, esquerda, superior, inferior e frontal e foto frontal neutra e sorrindo.

A base de dados utilizada nesse trabalho foi disponibilizada por um especialista em prótese dental e não está disponível publicamente. A base possui, no total, 1215 imagens. Das quais, 60 foram separadas para testes.

As Figuras 2 e 3 exemplificam o resultado da classificação, com sucesso da classe telerradiografia lateral.

A Figura 4 exemplifica, o resultado da classificação, com sucesso, da classe panorâmica.

A acurácia obtida, por meio dos testes com todas as classes da base de imagens, foi de $98,33 \%$.

\section{CONSIDERAÇÕES FINAIS}

Com base no estudo da literatura, bem como dos testes efetuados, foi possível identificar que técnicas de processamento de imagens e redes neurais convolucionais podem ser aplicadas para apoiar o especialista em odontologia em diversos tratamentos.

O protótipo atual teve como propósito inferir a qual classe a imagem pertence, sendo possível o incremento do mesmo para reconhecer elementos relacionados ao tratamento de prótese dentária, para apoiar a modelagem de próteses. Outro possível desdobramento do trabalho proposto, é o incremento do protótipo para solucionar demais problemas relacionados à área de odontologia.

Após o desenvolvimento do protótipo, o mesmo será avaliado por meio de métricas estatísticas como acurácia, precisão, revocação e

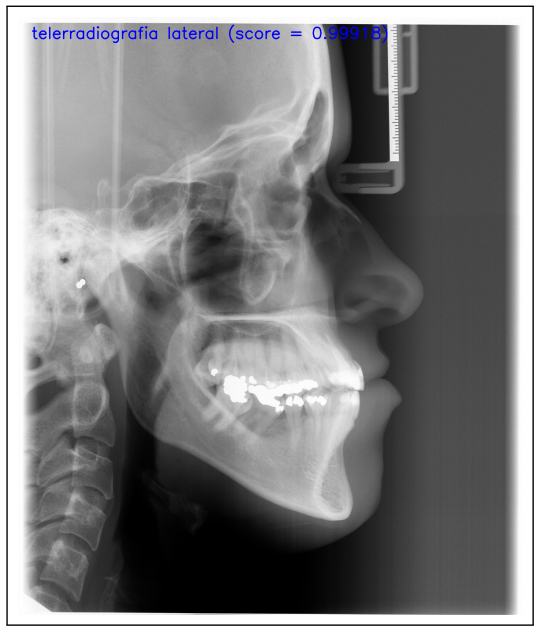

Figura 2: Classificação telerradiografia lateral - $1^{\mathrm{a}}$ amostra

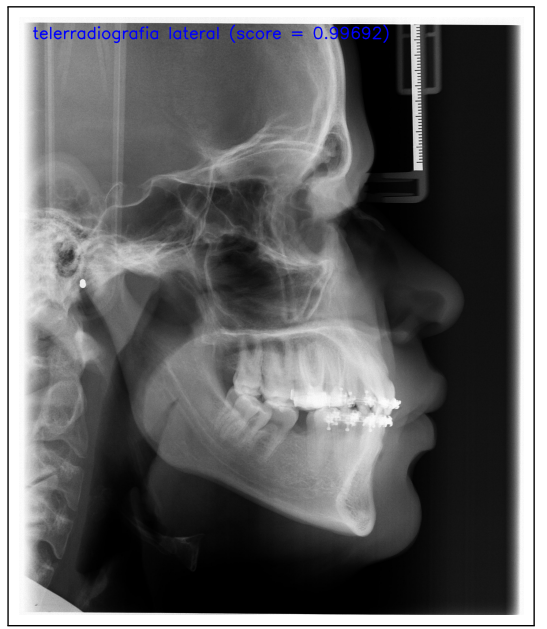

Figura 3: Classificação telerradiografia lateral - $2^{\mathrm{a}}$ amostra

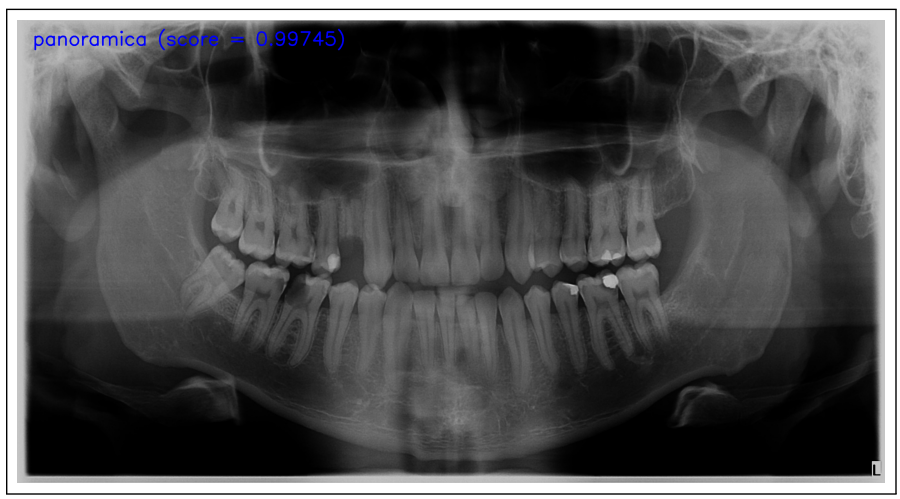

Figura 4: Classificação panorâmica - $1^{\mathrm{a}}$ amostra

medida F. Os resultados obtidos por meio da utilização do protótipo 
XII Computer on the Beach

desenvolvido serão comparados com os resultados da avaliação humana (ground truth).

\section{REFERENCES}

[1] Osmar Castro. Clonagem Terapêutica para Prótese totais e overdentures. Editora Santos, São Paulo: Santos, 2 edition, 2015. ISBN: 978-85-277-2696-2.

[2] Daniel de Moraes Telles. Prótese Total: Convencional e sobre implantes. Editora Santos, São Paulo: Santos, 2010. ISBN: 978-8572887564.

[3] Teruhiko Hiraiwa, Yoshiko Ariji, Motoki Fukuda, Yoshitaka Kise, Kazuhiko Nakata Akitoshi Katsumata, Hiroshi Fujita, and Eiichiro Ariji. A deep-learning artificial intelligence system for assessment of root morphology of the mandibular first molar on panoramic radiography. Dentomaxillofacial Radiology, 48(3):20180218, 2019.

[4] Joachim Krois, Thomas Ekert, Leonie Meinhold, Tatiana Golla, Basel Kharbot, Agnes Wittemeier, Christof Dörfer, and Falk Schwendicke. Deep learning for the radiographic detection of periodontal bone loss. Scientific reports, 9(1):1-6, 2019.

[5] Dmitry V Tuzoff, Lyudmila N Tuzova, Michael M Bornstein, Alexey S Krasnov, Max A Kharchenko, Sergey I Nikolenko, Mikhail M Sveshnikov, and Georgiy B Bednenko. Tooth detection and numbering in panoramic radiographs using convolutional neural networks. Dentomaxillofacial Radiology, 48(4):20180051, 2019.

[6] Dinggang Shen, Guorong Wu, and Heung-Il Suk. Deep learning in medical image analysis. Annual review of biomedical engineering, 19:221-248, 2017. 